\section{Cahiers de Narratologie}

Analyse et théorie narratives

$10.1 \mid 2001$

La voix narrative

\title{
Duras : Les voix du silence
}

\section{Madeleine Borgomano}

\section{OpenEdition}

\section{Journals}

Electronic version

URL: http://journals.openedition.org/narratologie/6945

DOI: 10.4000/narratologie.6945

ISSN: 1765-307X

\section{Publisher}

LIRCES

\section{Printed version}

Date of publication: 1 January 2001

Number of pages: $231-241$

ISBN: 2914561032

ISSN: 0993-8516

\section{Electronic reference}

Madeleine Borgomano, "Duras : Les voix du silence", Cahiers de Narratologie [Online], 10.1 | 2001, Online since 24 October 2014, connection on 23 February 2021. URL: http://journals.openedition.org/ narratologie/6945 ; DOI: https://doi.org/10.4000/narratologie.6945 


\title{
DURAS : LES VOIX DU SILENCE
}

\author{
Madeleine BORGOMANO
}

Université d'Aix-Marseille I

« Duras, les voix du silence ». Formule d'emprunt, déviée, déplacée, pour servir de titre. Mais faut-il le prendre "pour argent comptant »1 ce paradoxe à la Duras, détour par « une voix comme greffée, la voix d'un autre... $»^{2}$ ? Marguerite Duras le dit en d'autres mots dans Écrire ${ }^{3}$ (1993) :

«Écrire c'est aussi ne pas parler. C'est se taire. C'est hurler sans bruit » (É34).

Et pourtant, derrière cette écriture "du silence », quel bruissement de voix ! Au cinéma, d'abord. Car les « voix » durassiennes écrites doivent au cinéma beaucoup de leur étrangeté. Dans un entretien sur India Song4, à la question : "C'est quoi, les voix dans le film?», M. Duras répond : «C'est une polyphonie ». Elle énumère les voix des acteurs, celle des invités, "les voix enfouies I, les voix enfouies II. Et les voix perdues ", les voix 1 et 2 , «des jeunes femmes qui commentent la première partie du film, qui sont les voix d'une espèce de mémoire, ce que Blanchot appelle la mémoire de l'oubli $[\ldots]$ Et ensuite les voix 3 et $4[\ldots]$ celles des auteurs, des moteurs de l'histoire. Enfin «la voix qui lie toutes ces voix et qui est la voix de Viviane Forrester. Les voix des jeunes femmes, nos voix et celle de Viviane Forrester parlent

${ }^{1}$ Voir les considérations sur le titre, in Jacques Derrida, Donner le temps, Paris, Galilée, 1991, p. 109.

2 Marguerite DURAS, Le Vice-Consul, Paris, Gallimard, 1966, p. 131 (à propos du vice-consul).

${ }^{3}$ M. DURAS, Écrire, Paris, Gallimard, 1993. Marguerite Duras est morte le 3 mars 1996. Son dernier livre s'intitule C'est tout, Paris, P.O.L., 1995.

${ }^{4}$ M. DURAS, India Song (film), Distrib. Films Armorial, 1975. 
de l'histoire au passé. Tandis que les voix enfouies, les voix perdues, les voix présentes pendant la réception, en parlent au présent. $»^{5}$.

Le cinéma rend audible cette "polyphonie » complexe, silencieusement présente partout dans les livres, dès Un Barrage contre le Pacifique $(1950)^{6}$, et s'amplifiant au fil du temps, surtout à partir des années 70, pour, ensuite, progressivement s'épurer et s'épuiser. Les histoires racontées par Duras ne sont souvent que banales « histoires de quatre sous $»^{7}$. Mais les jeux de ces voix multiples : contrepoints et superpositions, entrelacements et emboîtements, mises en cause et mises en crises, les disloquent et les métamorphosent, ouvrant vertigineusement les gouffres du sens.

Je suivrai cette évolution dans l'œuvre en m'arrêtant sur deux livres très connus, l'un relevant de la fiction: Le ViceConsul (1965), l'autre de l'autobiographie (ou de la mythobiographie), $L^{\prime}$ Amant ${ }^{8}$ (1984) et sur quelques films.

\section{Le Vice-consul Au premier niveau, une voix démultipliée}

Au premier niveau narratif, Le Vice-consul suit, pendant deux jours, les activités mondaines d'un petit Cercle de Blancs de Calcutta, aimantés par deux pôles : l'attraction pour la très séduisante ambassadrice Anne-Marie Stretter et la répulsion pour le vice-consul "déplacé » (35) à Calcutta à la suite d'accès de violence, à Lahore. Aucune "histoire ", à part l'esclandre du vice-consul à la fin d'un bal et un voyage aux îles du delta. Mais « des histoires », des fragments d'histoires incertaines.

Pour raconter ces histoires exsangues, pas de narrateur. Ou plutôt rien d'autre qu'une "instance » abstraite, purement logique, qui pourtant se démarque nettement du narrateur

5 Nicole-Lise BERNHEIM, Marguerite Duras tourne un film, Paris, Albatros, 1981, p. 122.

6 M. DURAS, Un Barrage contre le Pacifique, Paris, Gallimard, 1950.

7 M. DURAS, Hiroshima, mon amour, scénario et dialogues, Paris, Gallimard, 1960, col. Folio, p. 118.

8 M. DURAS, L'Amant, Paris, ed. de Minuit, 1984. 
classique. Le récit, au présent, construit le leurre d'une simultanéité et d'une "présence " virtuelle, à la manière de celle des images d'un film. Cette non-voix ne se targue d'aucune prééminence, n'affecte aucune certitude et ne tente aucune remise en ordre des éclats disloqués qui lui parviennent en désordre. Sans cesse, elle se laisse interrompre et réduire au silence par le murmure d'autres voix plus ou moins anonymes. Parfois la voix virtuelle s'efface complètement pour laisser parler des personnages. Le vice-consul et le directeur du Cercle s'esquivent en un faux dialogue burlesque, racontant " des balivernes » (98) qui peuvent aussi bien s'échanger.

Le démembrement de la voix narrative culmine dans le long récit de la réception à l'Ambassade. Exclusivement descriptrice, elle ne reste pas neutre :

« Les ventilateurs plafonniers font un bruit d'oiseaux effarouchés, d'un envol immobile au-dessus de la musique, des lents fox-trot, des faux lustres, du creux, du faux, du faux or » (93).

Impersonnel et indéfini, le narrateur laisse percer une secrète ironie :

«- Entendez-vous crier?

- Ce sont des lépreux ou bien des chiens ? [...]

- J'ai confondu de loin, comme ça, à travers la musique, les aboiements des chiens et ceux des lépreux qui rêvent » (95).

Tout le reste transite par les voix confuses du " on", produisant l'effet de brouillage qui deviendra audible dans India Song. On parle du vice-consul : "Pourquoi l'a-t-elle invité ? " (93), de Lahore : "Mais qu'a-t-il fait au juste » Il a fait le pire, mais comment le dire ? » (94). On s'interroge sur Anne-Marie Stretter : "Elle intrigue, la femme de Calcutta » (93). «Personne ne sait ce qu'elle fait dans la villa des bouches du Gange. On dit que ses amants sont anglais [...] On dit que l'ambassadeur sait $[\ldots]$ Lorsqu'elle revient à Calcutta, sa vie très ponctuelle recommence [...] c'est ce qu'on voit. Et puis ? On ne sait pas » (96).

"On ne sait pas », " on » se demande et « on » invente. L'ignorance est au cœur du récit. Comme elle était déjà, 
explicitement, au cœur du Ravissement de Lol V. Stein, où Jacques Hold, le narrateur découvrait :

«[...] moi seul de tous ces faussaires, je sais : je ne sais rien

[...] ne rien savoir de Lol était la connaître déjà » (RLVS81)

Ainsi, le récit premier, en proie à toutes ces voix, reste " désaccordé " ${ }^{9}$, comme le piano d'Anne-Marie Stretter. Mais il se voit encore plus gravement mis à mal par le récit second qu'il englobe.

\section{Un récit miné par le récit même}

Mieke Bal, a étudié les relations entre ces deux récits, dès 1977 , dans « Hypo-récits ou la structure de la destruction » 10 . Sans entrer dans la polémique terminologique qui opposait les " hyporécits » aux " métarécits » de Genette"1, j'en resterai au terme d' "enchâssement » qui suffira pour désigner les rapports de subordination entre les deux récits qui alternent dans la première partie du roman.

Deux récits, donc deux voix narratives. On se souvient de l'incipit programmatique du roman : «Elle marche, écrit Peter Morgan ». Le narrateur premier, après trois mots seulement, cède la parole pour tout le début du livre, à ce jeune anglais assez inconsistant. Pourtant, vingt pages plus loin, la voix première, reprenant la narration, commente avec condescendance l'entreprise du " jeune homme » plein d'illusions, pour qui l'écriture est un moyen de connaissance et de salut :

«Peter Morgan est un jeune homme qui désire prendre la douleur de Calcutta, s'y jeter, que ce soit fait, et que son ignorance cesse avec la douleur prise » (29).

Il avouera d'ailleurs lui-même : « Je m'exalte sur la douleur aux Indes. Nous le faisons tous plus ou moins, non? » (157). «Le sanglot de l'homme blanc », en somme, avant la lettre.

9 «Le piano est désaccordé », répond Anne-Marie Stretter à ses amis. «Le piano n'est pas tellement désaccordé, c'est une phrase qui te plaît » (p. 161).

10 Mieke BAL, Narratologie, Paris, Klincksieck, 1977, p. 59-85.

11 Voir Mieke BAL, op. cit., p. 35, et G. GENETTE, Nouveau discours du récit, Paris, Seuil, 1983, p. 61. 
Pourtant, à travers ce narrateur second qui est aussi un apprenti écrivain, ce que le roman met en scène, c'est bien la fabrique de l'écriture. Elle naît d'une urgence personnelle : comme tous les blancs, Peter Morgan a "des Indes souffrantes » (157). Il " ne supporte pas » la misère de Calcutta : «Nid de fourmis grouillant [...] fadeur, épouvante, crainte de Dieu et douleur, douleur, pense-t-il » (30). « À bien écouter tout crie doucement mais loin, de l'autre côté du Gange » (151). L'écriture naît aussi de la fascination pour ce cri silencieux.

Peter Morgan a choisi une mendiante des rues de Calcutta, emblème du dénuement absolu, puisqu'elle a tout perdu, même la raison et la parole. Sur ce vide radicalement autre (femme, indienne et misérable), et à l'aide d'un souvenir d'Anne-Marie Stretter (la « vente » d'une enfant (72)), le jeune anglais « invente » :

«Peter Morgan voudrait maintenant substituer à la mémoire abolie de la mendiante le bric-à-brac de la sienne [...] Quoi dire à la place de ce qu'elle n'aurait pas dit ? de ce qu'elle ne dira pas? de ce qu'elle ignore avoir vu ? de ce qu'elle ignore avoir eu lieu ? à la place de ce qui a disparu de toute mémoire ? " (73).

L'histoire de la mendiante est animée par l'injonction de la mère qui a chassé sa fille enceinte : «Il faut se perdre ». Et la narration de cette histoire trouve son impulsion dans cette série d'interrogations angoissées et cette insondable ignorance. Mais en même temps, elle s'en voit contaminée. Le «Elle marche, écrit Peter Morgan » du début, se change en « Elle marcherait, dit-il » (179). Entre les deux, où est passé le livre ? Ne s'est-il pas, lui aussi perdu ? Tous les jeunes amis d'Anne-Marie Stretter, les élus du «saint synode de la blanche Calcutta»(152), viennent au secours de Peter Morgan, chœur polyphonique d'écrivains virtuels. "Moi, si j'en parlais, je lui ferais faire des choses à l'envers [...] » (181). Cependant, ils n'écrivent pas, ils parlent seulement et leur discours en appelle à Anne-Marie Stretter, seul ancrage « réel » de tant de fictions. Mais elle leur refuse sa caution. Le narrateur le répète avec insistance (neuf fois !), ponctuant leur discours, « elle se tait », « elle dort» (179-183). «Pourquoi 
parler à cette femme qui dort ? Discours inutile et silence profond » (182).

Toute l'entreprise d'écrire, au miroir de cet échec, se voit renvoyée au néant ; même celle du roman qui lui, pourtant, existe. D'ailleurs, comme le montrait déjà si bien Mieke Bal, en analysant l'effet destructeur des analogies troublantes entre les deux histoires :

«La structure narrative, si nette, si rassurante au début, signifie sa propre impossibilité [...] de la même manière que la structure coloniale signifie son auto-destruction. La destruction, comme la lèpre, est contagieuse : les deux récits s'infectent l'un l'autre $»^{12}$.

Marguerite Duras écrira, dans L'Amant:

«Quelquefois je sais cela : que du moment que ce n'est pas, toutes choses confondues, aller à la vanité et au vent, écrire ce n'est rien » (A15).

\section{L'effet-cinéma}

Dans les années 70, Marguerite Duras privilégie le cinéma dont elle use " pour détruire ce qui est écrit $»^{13}$, mais dont elle tire aussi une écriture subordonnée. L'écriture circule du livre au film et du film au livre, dans tous les sens. Et la voix narrative s'en trouve profondément transformée. Voyons ce qui se passe dans Le camion, en 1977. Les images du film font alterner des plans sur un camion bleu, circulant sur des routes de banlieue, et des plans sur une " chambre noire " (11) où Marguerite Duras et Gérard Depardieu lisent les feuillets d'un script. La fonction narrative, dont l'image est privée, est exclusivement accordée aux "voix » (à entendre au sens propre). Ces voix se trouvent à la fois très visiblement « incarnées » et arrachées à leur référence évidente. L'auteur et l'acteur, déplacés, jouent bien tous deux un rôle, mais pas leur rôle dévolu. Ils jouent le seul rôle jamais représenté d'ordinaire, celui du lecteur. Troublés, ils sèment le trouble. Qui parle?

12 Mieke BAL, op. cit., p. 82.

13 M. DURAS, jaquette de La femme du Gange, Paris, Gallimard, 1973. 
La voix de Marguerite Duras se démultiplie : voix off, ouvrant le film « Ç'aurait été une route » (10), elle renvoie à l'écrivain mais aussi à ces "voix de l'invisible » qui parlent dans les films, venant d'on ne sait où. Puis, voix de Duras metteur en scène, parlant avec son acteur, Depardieu : "Ç'aurait été un film », elle use de son autorité pour déréaliser le film, et sa propre voix, par l'usage de ce conditionnel irréel du passé. Puis elle devient voix de la lectrice, reproduisant la voix du personnage de la vieille dame auto-stoppeuse, sans pourtant rechercher l'illusion de réalité, puisque le bavardage de la femme est distancé par l'insistance des « elle dit».

Sans m'attarder davantage sur cette déconstruction, je constaterai que la voix narrative, dans le filtre du film, se trouve à la fois simplifiée (on n'entend plus que deux voix) et réincarnée, mais dans le même temps, déstructurée, éclatée.

Dans L'Homme Atlantique ${ }^{14}$, la simplification continue, le démembrement aussi. On n'entend plus qu'une seule voix, celle de Duras, mais désincarnée, résolument « off ». Cette voix unique accompagne un film-limite, qui, à part quelques échappées maritimes et quelques vues d'un homme presque immobile, ne montre qu'un écran noir. La voix assume son statut de maîtresse du film. Elle s'adresse essentiellement à un « vous » (peut-être le personnage montré), comme à un acteur. "Vous ne regarderez pas la caméra », tels sont ses premiers mots. Prescriptive, mais négative, elle ordonne les non déplacements de sa marionnette: "Vous êtes le seul à tenir lieu de vous-même auprès de moi dans ce moment-là du film qui se fait » (10). Mais insensiblement, tandis que l'homme « sort du champ de la caméra » (15), la voix se met à raconter sa propre histoire de séparation et d'absence : «j'ai dit à voix haute la date du jour [...] le lundi quinze juin 1981, que vous étiez parti dans la chaleur terrible pour toujours » (19). Puis elle revient au film, à sa genèse : "Vous êtes resté dans l'état d'être parti. Et j'ai fait un film de votre absence » (22). Elle reprend alors ses commandements : "Vous allez repasser de nouveau devant la caméra. [...] Ne bougez pas. [...] Oui, ça va recommencer » $(22)$

14 M. DURAS, l'Homme Atlantique, film, 1981, prod. Berthemont, et récit, Paris, Minuit, 1982. 
Ainsi se départage la voix en apparence unique, qui a glissé du conditionnel à l'impératif et à l'optatif, pour tenter de dire dans un film bouleversant l'absence, la mort et le désir.

\section{L'Amant}

L'Amant, en 1984, revient à l'écriture. Mais la voix très particulière qui s'y manifeste doit beaucoup à cette longue transition cinématographique.

L'écriture de l'Amant, plus ou moins autobiographique, est dominée par la première personne. Le « je » autobiographique télescope les références, puisqu'il renvoie non seulement au personnage et au narrateur, mais encore à l'auteur même, ce qui ne laisse pas malgré les apparentes évidences, de poser quelques problèmes. Car ces trois «instances» sont supposées fondues dans l'identité d'une seule "personne » (extradiégétique, évidemment), stable malgré le temps et l'espace.

\section{Le présent du désir}

L'Amant, tout en en conservant la nostalgie, fait éclater cette identité (unicité) illusoire. Dès l'incipit : «Un jour, j'étais âgée déjà $[\ldots]$ », la " parleuse » souligne qu'elle n'a plus quinze ans depuis longtemps. Elle insiste tout au long d'une page sur son visage « lacéré de rides [...] à la peau cassée. [...] J'ai un visage détruit » $(A 10)$. Or la phrase qui succède, sans transition aucune, à ce constat de défaite, opère un bond prodigieux dans le temps : «Que je vous dise encore, j'ai quinze ans et demi ». Brusquement, le « je », changeant complètement de référence, évoque "la petite au chapeau de feutre ", sur le bac qui traverse le Mékong. L'emploi du présent forge une fusion virtuelle entre les identités et les espaces-temps.

Bien sûr, le lecteur tend à édulcorer le scandale. Au présent de «j'ai quinze ans et demi » (et à tous les autres du même type qui suivront), il ôte sa valeur déictique pour l'interpréter comme une sorte de prosopopée, une figure rhétorique du désir. Mais lisons à la lettre.

L'écriture, en exploitant la magie du langage, tend à remonter le temps et à abolir ses ravages : il suffit, en somme, de dire « je » et d'employer le présent pour que la vieille narratrice et 
l'enfant au visage lisse ne fassent plus qu'une. Mais les rétroprojections du "vrai » présent abîment l'image ancienne : "Maintenant je vois que très jeune, à dix-huit ans, à quinze ans, j'ai eu ce visage prémonitoire [... ] J'avais à quinze ans le visage de la jouissance » $(A 15)$. Et surtout, les fluctuations permanentes des personnes et des temps dévoilent l'instabilité de l'illusion, fragile comme un reflet sur l'eau du fleuve. Les fragments morcelés de ce texte déstabilisent les repères identitaires, autant que les repères spatio-temporels.

Au présent de l'énonciation, jouant normalement avec des passés dits composés, mais dont l'effet serait plutôt de "décomposer ", et au présent qui recrée le temps perdu de l'histoire amoureuse («Les baisers sur le corps font pleurer. On dirait qu'ils consolent » (58)), se juxtaposent des présents renvoyant à d'autres périodes de la vie, à d'autres lieux, la vie et la mort des frères, de la mère, Paris pendant l'occupation (82). Se mêlent encore des présents beaucoup plus généraux : " écrire, ce n'est rien" (15). D'autres que l'on pourrait nommer des présents de l'image "L'image dure pendant toute la traversée du fleuve » (11). Présents aussitôt ébranlés par un conditionnel irréel du passé : "L'image [...] aurait pu exister » (16), mais « elle n'existe pas. Elle a été omise. Elle a été oubliée " (17). À quoi s'ajoutent d'étranges futurs, sur la mort du père, par exemple, (qui a eu lieu quand Marguerite Duras avait sept ans 15) : «Il mourra dans moins d'un an. Ma mère aura refusé de le suivre en France » (41).

\section{Un « je » éclaté}

Ces dérapages temporels se doublent de troublantes fluctuations pronominales. Parfois, l'usage du «je » et du présent donne à la "petite au chapeau de feutre " un statut de narratrice, en supprimant la vieille femme intermédiaire : " Je descends du car. Je vais au bastingage. Je regarde le fleuve » (17). D'autres fois, dans une même phrase, elles se partagent le rôle: "Quand je suis sur le bac du Mékong, ce jour de la limousine noire, la concession du barrage n'a pas encore été abandonnée par ma mère » (35). Tandis que la jeune fille vit au

${ }^{15}$ En 1921. Marguerite Duras est née en 1914. 
présent la traversée du fleuve, son avenir est en marche et l'autre le sait et dit qu'elle le sait.

Pourtant, dans les scènes les plus intenses, celle de la rencontre, celle de la garçonnière, la petite cesse d'être sujet du discours et se voit reléguée à la troisième personne, celle de « l'absente » (selon la classification de la grammaire arabe), la «non-personne », selon Benveniste. Elle se trouve alors éloignée, projetée dans la distance de la fiction, mais non sans déchirement, dans la contradiction d'une présence de l'absence, proche parente de « la mémoire de l'oubli ».

Ainsi, l'unicité d'un apparent monologue éclate-t-elle en voix multiples disloquées par le temps, mais rêvant sans cesse d'une impossible fusion.

\section{Un pressant appel au lecteur}

Cette voix narratrice éclatée peut bien décontenancer le lecteur, elle n'en lance pas moins un appel pressant à son écoute et à sa bienveillance.

«Que je vous dise encore... » (11), « oui, que je dise » $(37,94)$. Ou bien, plus audacieusement : «Sur le bac, regardez-moi, je les ai encore. Quinze ans et demi » (24). On sait que L'Amant a d'abord été conçu comme légendes pour un album de photographies. Mais aussi que cette photo-là, celle du bac, n'existe pas. « [c]'est à ce manque d'avoir été faite qu'elle doit sa vertu, celle de représenter un absolu » (17). La sollicitation en devient beaucoup plus radicale. Le lecteur se trouve, bon gré, mal gré, entraîné par la narratrice et avec elle dans ce monde d'absence. En fait, selon la stricte théorie narratologique, seule cette instance abstraite et forgée par le texte, « le narrataire » se voit ainsi sollicitée. Le lecteur, lui, actualisation " réelle » de cette instance, pourrait bien résister à l'appel de la sirène. Mais pas si facilement. Car ce ton de confidence murmurée a quelque chose d'irrésistible.

\section{Des voix qui naissent du silence,}

Telle sera désormais la voix narratrice des derniers livres. Elle a acquis dans les films son statut un peu fantastique de voix " off ", brouillant le dehors et le dedans, à la fois impersonnelle et très personnelle, lointaine et pourtant très proche, absente 
et si fortement présente, elle n'a cependant plus rien de l'abstraction du narrateur classique. Remontant en arrière, elle reconstitue plutôt les conditions du conteur en se forgeant un lecteur-auditeur " complice, forcément complice » à qui elle murmure son histoire. Toujours la même histoire, surgie de la fascination du silence, autour d'un être impénétrable, refermé autour de son secret, qui, comme le vice-consul de Lahore " vous force à penser à lui » (VC153). Le plus souvent, une de ces femmes "définitivement perdues, absolument, même à elles-mêmes " ${ }^{16}$, qui peuplent l'univers durassien, Lol V. Stein ou Anne-Marie Stretter, la mendiante ou Émily L.

Mais cette voix née du silence le brise à peine. Ses doutes, ses ignorances et ses incertitudes le laissent se refermer autour d'elle, l'absorber peu à peu.

Voix du silence, ancrée dans la "mémoire de l'oubli », elle retourne finalement, au silence vers lequel elle a toujours tendu. « La polyphonie [...] cela rend la mort présente peutêtre » disait Marguerite Duras dès 197517.

16 M. DURAS à Montréal, Montréal, édition Spirale, 1981, p. 40.

${ }^{17}$ Marguerite Duras tourne un film, Paris, Albatros, 1975, p. 123. 